\title{
Noise canceling to compute second order statistic from SPIV
}

\author{
J-M. Foucaut ${ }^{1 *}$, R. Yuvaraj ${ }^{1}$, C. Cuvier ${ }^{1}$ \\ ${ }^{1}$ Univ. Lille, CNRS, ONERA, Arts et Métiers ParisTech, Centrale Lille, UMR9014-LMFL-Laboratoire de \\ Mécanique des Fluides de Lille-Kampé de Fériet, F-59000, Lille, France, Lille, France \\ * jean-marc.foucaut@centralelille.fr
}

\begin{abstract}
Stereoscopic PIV (SPIV) is performed near the wall in a turbulent boundary layer at high Reynolds numbers $\left(R e_{\tau}=2272,3840\right)$ in the streamwise-wallnormal plane. A novel method for denoising the statistics is proposed by the use to two independent SPIV systems to capture the same field of view, with the objective being that the random noise associated with the velocity fluctuations are not correlated between two independent PIV systems. The derivatives in the third (spanwise) direction are obtained by axisymmetry assumptions [George and Hussein (1991)] and continuity equation. The statistics are in agreement with that of DNS at comparable Reynolds number, from $y^{+}=25$ for lower $R e_{\tau}$ experiment and from $y^{+}=40$ for higher $\operatorname{Re}_{\tau}$ experiment.
\end{abstract}

\section{Introduction}

Stereoscopic PIV (SPIV) is a well recognized experimental method to study turbulent flow. Many researchers also use this method to compute statistics of the flow such as mean velocity, Reynolds stress tensor, probability density function or spectra [Adrian et al. (2000), Foucaut et al. (2011), Herpin et al. (2013)]. SPIV allows the measurement of the three components of the velocity in a plane with an accuracy of about 1-2\% ( 0.1 pixel). For a turbulent flow, it opens up the unique capability of studying the organization of the turbulence. Generally, due to the limited spatial resolution of PIV, the smallest scales of the flow that can be investigated is limited. The PIV random noise will also affect the statistics such as variance. In addition there is also the noise amplification that occurs when derivatives are computed [Foucaut and Stanislas (2002)]. Nevertheless, the velocity gradient is necessary to determine the vorticity, the shear, the dissipation and specific criteria allowing the detection of vortices such as the Q criterion which are useful tools to study the flow organization.

In the present contribution we focus on statistics. The proposition here is to build an experiment of time resolved SPIV in the near wall region of a turbulent boundary layer with a very good spatial and temporal resolution. This experiment is carried out in the LMFL boundary layer wind tunnel. It is setup with two SPIV system in order to be able to characterize and to get rid of the noise. The measured velocity is considered $u_{i}=u_{i_{T}}+u_{i_{N}}$ where $u_{i_{T}}$ is the true value and $u_{i_{N}}$ is the noise (random error). The variance, then computed by $\left\langle u_{i} u_{i}\right\rangle=\left\langle u_{i_{T}} u_{i_{T}}\right\rangle+\left\langle u_{i_{N}} u_{i_{N}}\right\rangle$ where \langle\rangle corresponds to averaging in time and homogeneous direction (in streamwise direction for the present experiment), is affected by the variance of the noise and thus it could be overestimated. The solution is to use 2 independent PIV systems to measure the same velocity at the same point. In that case the variance is computed as

$$
\begin{gathered}
u_{i_{1}}=u_{i_{T}}+u_{i_{N_{1}}} \\
u_{i_{2}}=u_{i_{T}}+u_{i_{N_{2}}} \\
\left\langle u_{i_{1}} u_{i_{2}}\right\rangle=\left\langle u_{i_{T}} u_{i_{T}}\right\rangle
\end{gathered}
$$

where the numbers 1 and 2 correspond to each PIV system. The variance is free of noise because the noise of the two systems are not correlated. This allows the computation of denoised statistics and an estimation of the RMS value of the noise by

$$
\sigma_{u_{N}}^{2}=\left\langle u_{i_{1}} u_{i_{1}}\right\rangle-\left\langle u_{i_{1}} u_{i_{2}}\right\rangle
$$




\section{Experimental setup}

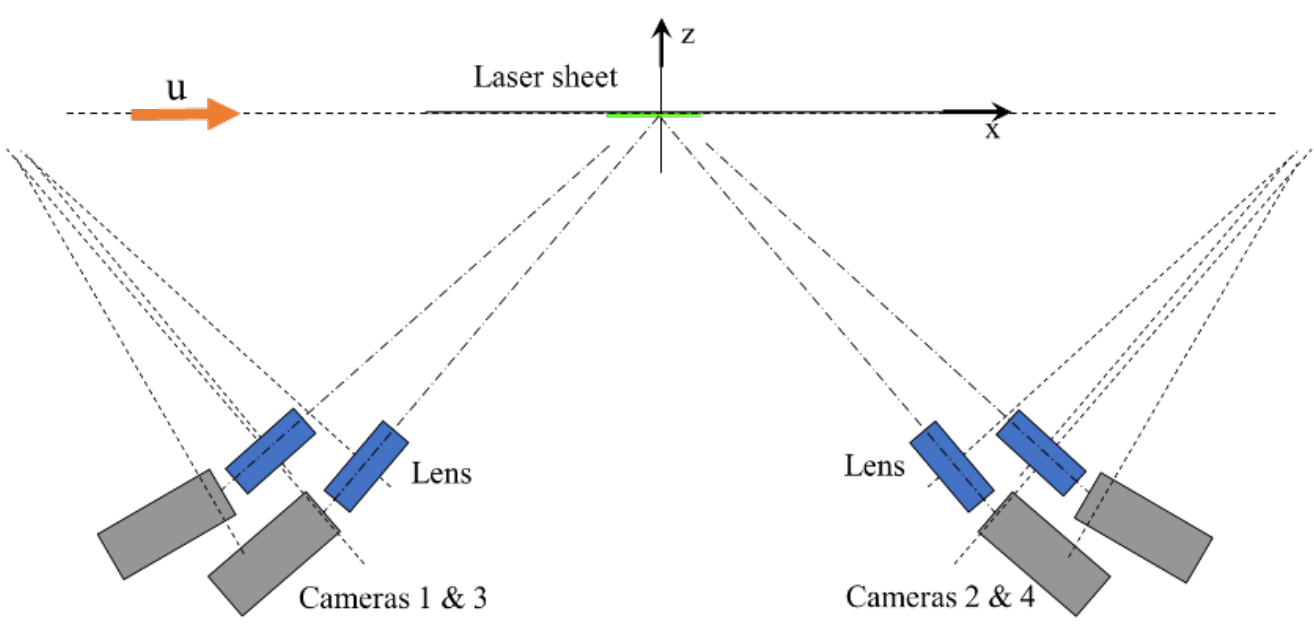

Figure 1: Schematic of the SPIV experimental setup

The measurement was done at $19.2 \mathrm{~m}$ from the inlet of the test section. At this station, the boundary layer thickness is of $0.273 \mathrm{~m}$ and $0.243 \mathrm{~m}$ for the Reynolds numbers $R e_{\tau}$ of 2220 and 3840 for infinite velocities of $3 \mathrm{~m} / \mathrm{s}$ and $6 \mathrm{~m} / \mathrm{s}$, respectively. Figure 1 shows the two sets of Stereoscopic PIV (2D-3C) employing 4 Miro cameras to capture a field of view illuminated by a laser light sheet of $60.4 \times 18.4 \mathrm{~mm}^{2}\left(503^{+} \times 153^{+}\right)$ for $R e_{\tau}=2220$ and $29.6 \times 18.4 \mathrm{~mm}^{2}\left(493^{+} \times 306^{+}\right)$for $R e_{\tau}=3840$, both along the streamwise $\mathrm{x}$ and wall-normal direction y. The seeding for the experiment is $1 \mu \mathrm{m}$-size water-ethylene glycol mixture which is fully seeded in the wind tunnel. The cameras and the Laser are synchronized by LaVision's High Speed Controller. The chip-size of the camera were reduced at $1280 \times 512$ pixels to achieve $4.5 \mathrm{kHz}$ PIV frequency and $640 \times 512$ pixels corresponding to $7.5 \mathrm{kHz}$ for the lowest and highest Reynolds numbers, respectively and the lasers are triggered at same time with $10 \mathrm{~mJ} / \mathrm{pulse}$. The images were acquired in a series of 5 images burst mode, up to a total of 1803 sets of time series images in order to offer possibility to make statistics on time derivative. This time-resolved SPIV employed here is capable of producing a 2D-3C velocity field in a XY-plane, which provides all the derivatives in $\mathrm{x}$ - and $\mathrm{y}$ - directions and time. With the help of continuity equation, it is possible to obtain the $\partial w / \partial z$ term. The experiment is calibrated by using an accurate calibration plate which has crosses at known places and are spaced equally by $1 \mathrm{~mm}$ distance, and this is in-turn captured by the cameras in 11 spanwise positions. The Soloff method is used for the threecomponent reconstruction. This calibration procedure is followed by self-calibration [Wieneke (2008)]. Special care is taken to compute the velocity vectors at the same point for the two SPIV system. To ensure that the velocities are computed at the same point the mesh is generated in the object plane and projected in the planes of the 4 cameras. The acquired data are then processed using a modified version of MatPIV toolbox at LMFL. A multi-pass cross-correlation PIV analysis is used with deformation at the last pass and with a final interrogation window size of $18 \times 24 \mathrm{px}$ which corresponds to $0.97 \times 0.97 \mathrm{~mm}^{2}$ for the system 1 and $0.88 \times 0.95 \mathrm{~mm}^{2}$ for the system 2, which are of the same order of magnitude corresponding to about $8^{+}$for $R e_{\tau}=2220$, and $16^{+}$for $R e_{\tau}=3840$. The small variation come from a little difference of angles and magnifications between the two systems.

Figure 2 shows the mean velocity profile in wall-units in SPIV experiment at the two $R e_{\tau}$, and is then compared with DNS of TBL at $R e_{\tau}=1989$ [Borrell et al. (2013)] and TCF at $R e_{\tau}=4200$ [Hoyas and Jiménez (2008)]. The ranges of accessible wall-distances from the SPIV experiment are $10.25 \leq y^{+} \leq 157.13$ (green vertical line) and $20.19 \leq y^{+} \leq 309.6$ (red vertical line), for the lower and higher $R e_{\tau}$ datasets respectively. The mean velocity $\left(\bar{u}^{+}\right)$of the SPIV experimental dataset at both the Reynolds numbers shows a good agreement that of the DNS datasets.

Figure 3 shows the comparison of variances computed from individual systems and denoised with that of DNS datasets at nearest $R e_{\tau}$. It is observed that the statistics of both the individual PIV systems and denoised version from $R e_{\tau}=2220$ follows that of the DNS at $R e_{\tau}=1989$ [Borrell et al. (2013)]. However, as the wall 


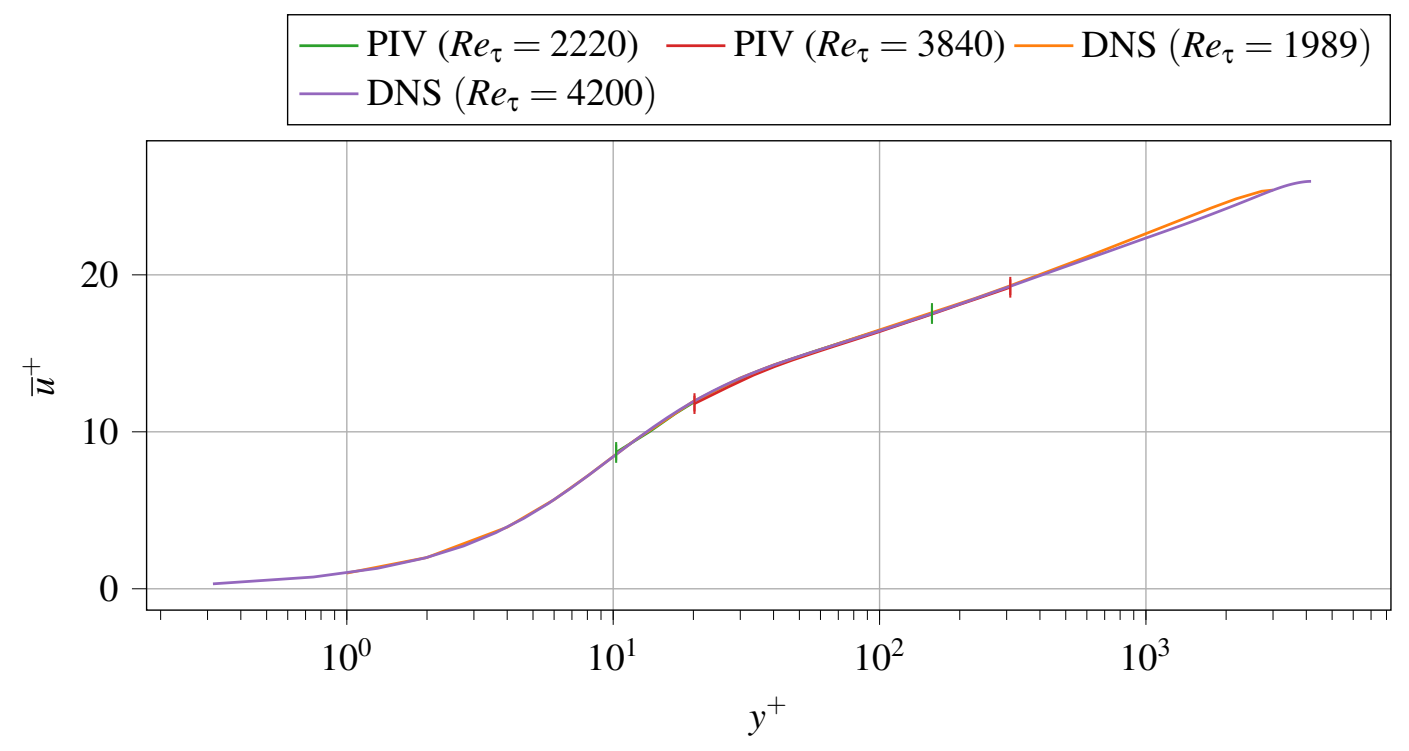

Figure 2: Comparison of average streamwise velocity normalised with inner co-ordinates $\bar{u}^{+}$from the present PIV experiments at $R e_{\tau}=2220$ and 3840 with DNS datasets of TCF at $R e_{\tau}=1989,3000,4200$. The green vertical line shows the range of wall-distances for PIV experiment with $R e_{\tau}=2220$, and the red vertical line shows the range of wall-distances for PIV experiment with $R e_{\tau}=3840$

is approached one can observe that only the denoised statistics tend to follow that of DNS down to $y^{+}=10$. This result shows the advantages of using two SPIV systems with the order of spatial resolution in present experiment for accurate measurement near the wall. The SPIV with higher $R e_{\tau}=3840$ does approximately follow that of DNS of TCF at $R e_{\tau}=4200$ [Hoyas and Jiménez (2008)] for $u^{\prime}, v^{\prime}$ and $u^{\prime} v^{\prime}$, and there is some discrepancy for $w^{\prime}$. It is clear that the noise is very low in the present experiment, with the exception for the $\mathrm{w}$ component it is difficult to distinguish the noise.

Figure 4 gives a field of an estimation of RMS value of the noise associated with the two systems for the streamwise component in pixels. It is observed that with the exception on the left side of the fields and near the wall, the noise is of the order of 0.06-0.08 px. The higher value on the left side is probably linked to a problem of vector validation at the border.

Figure 5 shows the same parameter but averaged along $\mathrm{x}$ for both SPIV systems in pixels for $R e_{\tau}=2220$. The noise of the two systems stay under that $0.06 \mathrm{px}$ which is remarkable. It is compared with the model of George and Stanislas (2020) (denoted as 'Estimation George') which is able to estimate the noise by taking into account the pixelisation error and the fluctuation of particle positions in the interrogation window due to the local turbulence. This noise from 'Estimation George' is given by :

$$
\sigma_{t h}=\sqrt{\frac{1}{N}\left(\left\langle u_{1}^{\prime 2}\right\rangle^{v o l}+\frac{\Delta^{2}}{12}\right) W_{1}\left(U \delta_{t}\right)}
$$

where the first part of the right hand side of the equation correponds to the velocity fluctuation inside interrogation volume and the second part corresponds to the pixelisation error $(\Delta=0.5)$ and $\mathrm{N}$ is the number of particles in the interrogation window. It is also compared with an estimation from spectral analysis as in Foucaut et al. (2004) at distances of $45^{+}$and $100^{+}$. The noise from George and Stanislas (2020) is smaller compared to the noise from the present SPIV experiment, which therefore shows that other sources of noise needs to be accounted in addition to the pixelisation error and fluctuation of particle positions. This model does not take into account other source of noise such as isolated particles or gradient in the interrogation window. The estimation from the spectra [Foucaut et al. (2004)] is approximately of the same order as the noise estimated from the experiments. The spectra of the two systems and the subsequent noise estimation from them are very similar and of the same order as predicted by Equation 4.

Figure 6 shows the dissipation which is composed by a sum of variances of spatial derivative of velocity, 


\begin{tabular}{|c|c|c|}
\hline $\begin{array}{l}- \text { PIV } \operatorname{sys}_{1}\left(\operatorname{Re}_{\tau}=2220\right) \\
-\mathrm{PIV} s_{2}\left(\operatorname{Re}_{\tau}=2220\right) \\
-\mathrm{PIV} \text { denoised }\left(\operatorname{Re}_{\tau}=22\right.\end{array}$ & $\begin{array}{l}- \text { - PIV } \operatorname{sys}_{1}\left(\operatorname{Re}_{\tau}=3840\right) \\
-- \text { PIV sys } 2\left(R e_{\tau}=3840\right) \\
\text { - - - PIV denoised }\left(\operatorname{Re}_{\tau}=38\right.\end{array}$ & $\begin{array}{r}-\operatorname{DNS}\left(R e_{\tau}=1989\right) \\
-\operatorname{DNS}\left(R e_{\tau}=4200\right)\end{array}$ \\
\hline
\end{tabular}
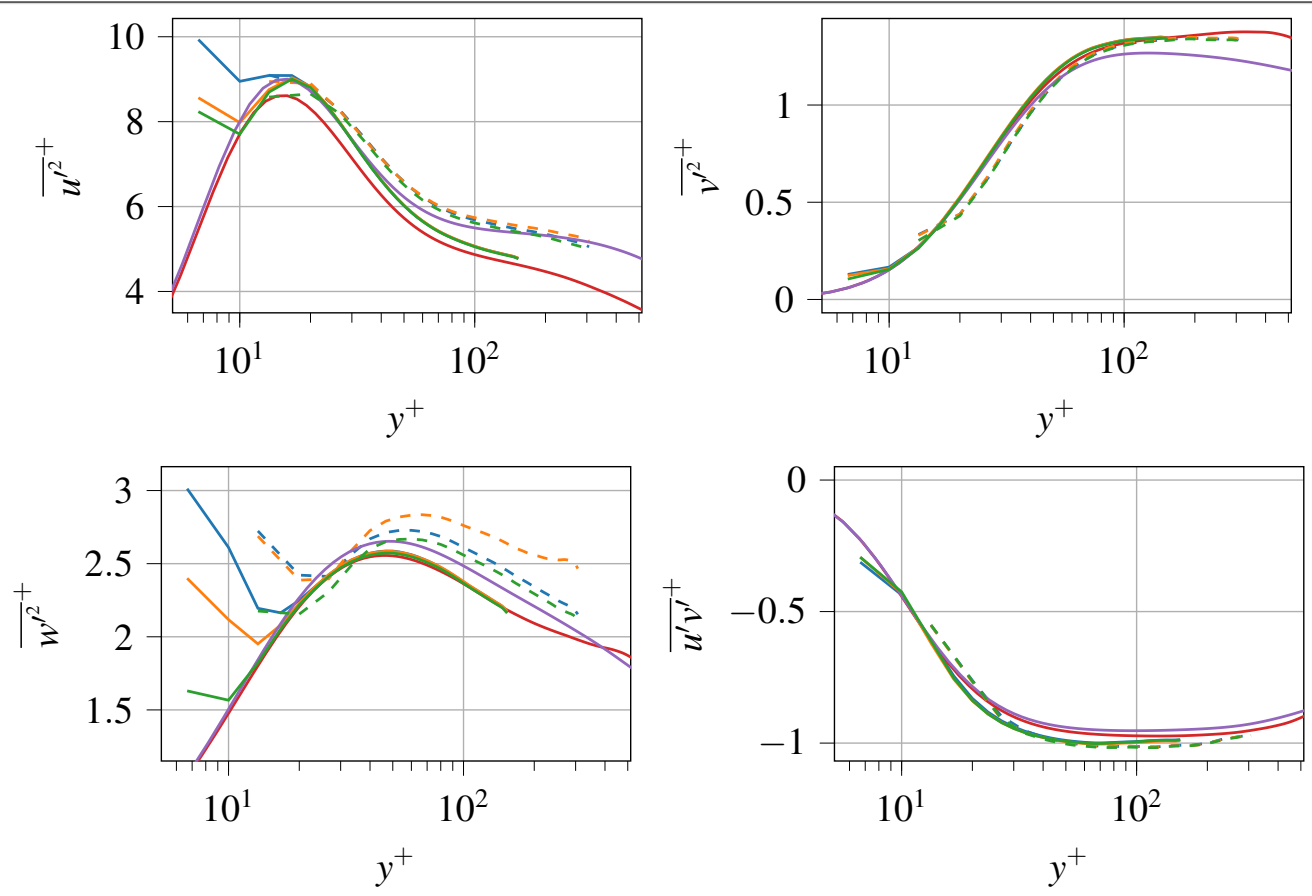

Figure 3: Comparison of variances and covariances of streamwise, wall-normal and spanwise velocity components in the present PIV experiments at $R e_{\tau}=2220$ and 3840 with DNS of TCF datasets at $R e_{\tau}=1989,3000$ and 4200
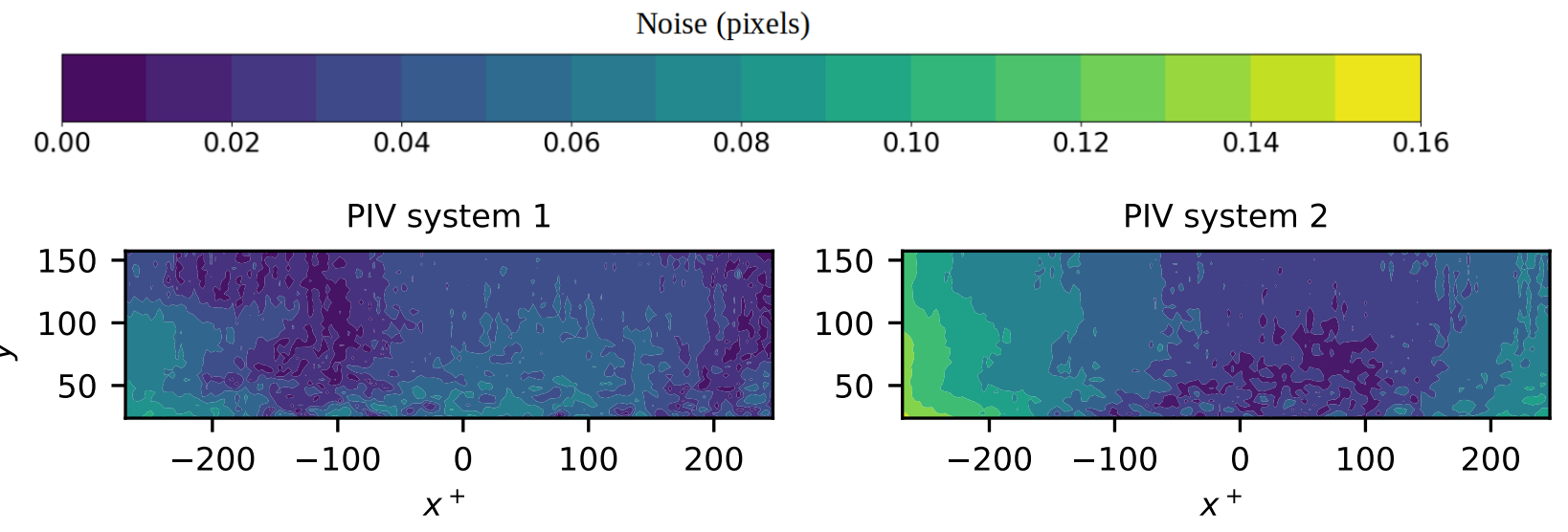

Figure 4: Noise associated with SPIV $\operatorname{sys}_{1}$ (left) and $s s_{2}$ (right) in pixels

obtained with individual system and denoised by the two systems. The derivatives are computed by a Least Squared Method [Foucaut and Stanislas (2002)] which is based on second-order central difference scheme and is optimized to not amplify the noise. The missing variance of derivatives are obtained by the assumption of axisymmetry (see George and Hussein (1991)). We can see the noise which is very small except close to the wall, and the effect of filtering for the highest Reynolds Number. This allows to evidence the filtering effect of PIV for the highest Reynolds number [Atkinson et al. (2014)] which is mainly visible in the dissipation rate. The assumption of axisymmetry works down to $y^{+}=25$ for the SPIV experiment at 


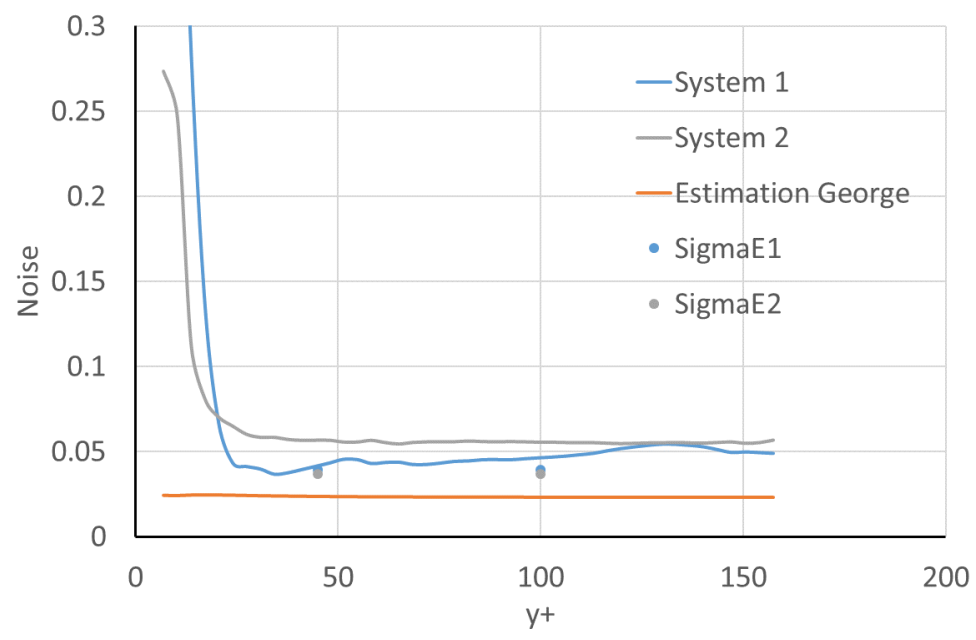

Figure 5: Noise associated with SPIV $s_{1} s_{1}, s_{s_{2}}$ in pixels, compared with the noise estimation of George and Stanislas (2020), and that of estimation from spectra Foucaut et al. (2004)

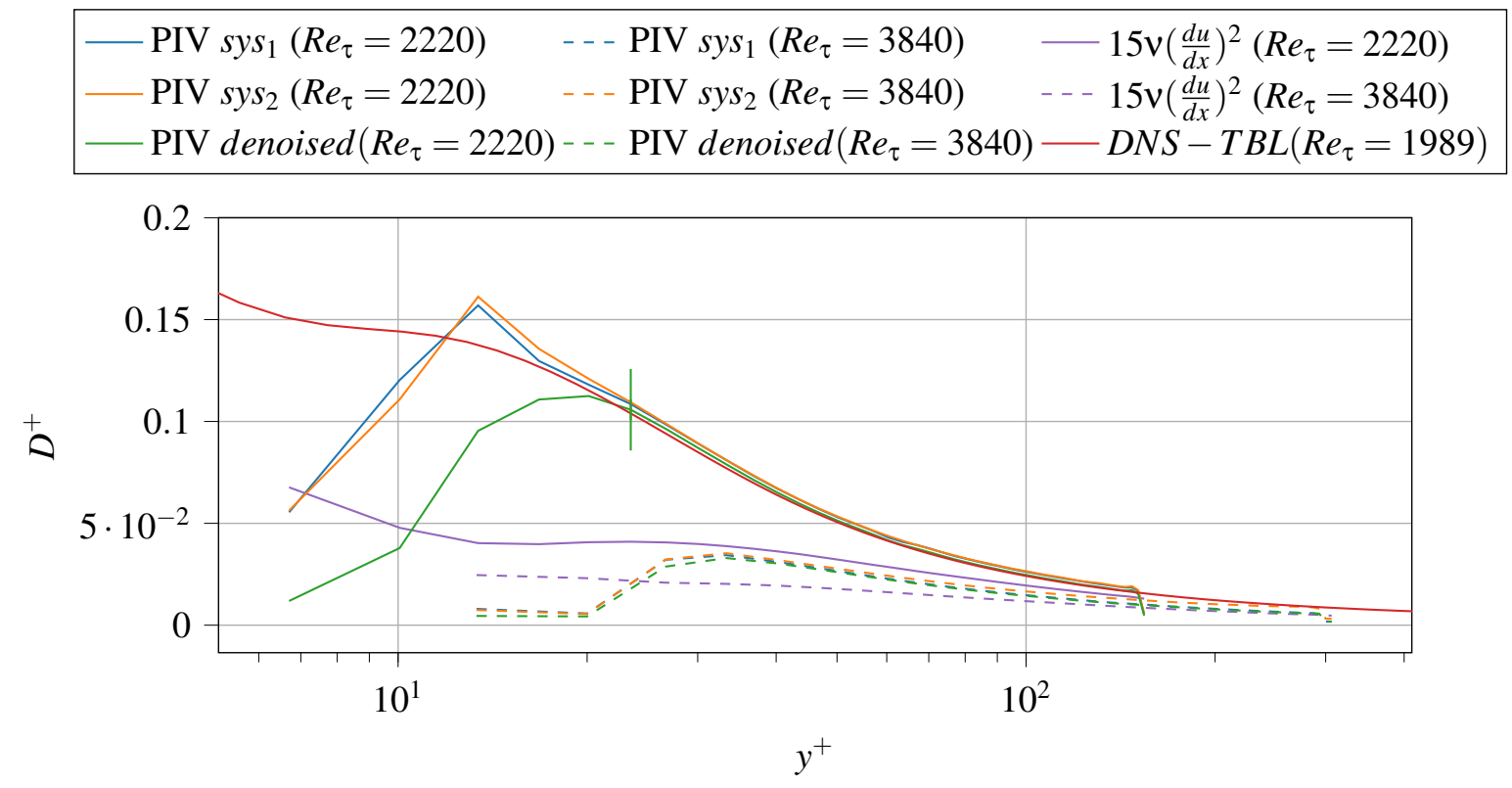

Figure 6: Comparison of dissipation computed from individual PIV systems 1, 2 and denoised at $R e_{\tau}=2220$ and 3840 with DNS of TBL datasets at $R e_{\tau}=1989$. Vertical green line shows the limit of axisymmetric assumption in the computation of dissipation

$R e_{\tau}=2220$ which follows the explanation in Foucaut et al. (2020).

Figure 7 shows the effect of denoise on the streamwise derivatives of two velocity components obtained from SPIV experiment at $R e_{\tau}=2220$. It is observed for ${\overline{\left(\frac{\partial u^{\prime}}{\partial x}\right)^{2}}}^{+}$, the difference between the individual systems

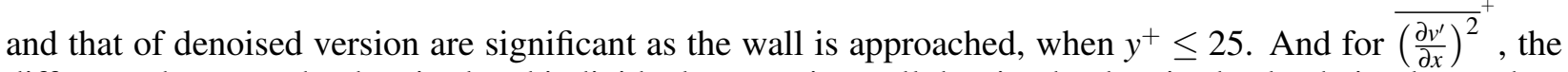
difference between the denoised and individual system is small despite the denoised value being lower than that predicted by individual systems. So this method of denoising has the ablity to obtain accurate statistics especially near the wall. 


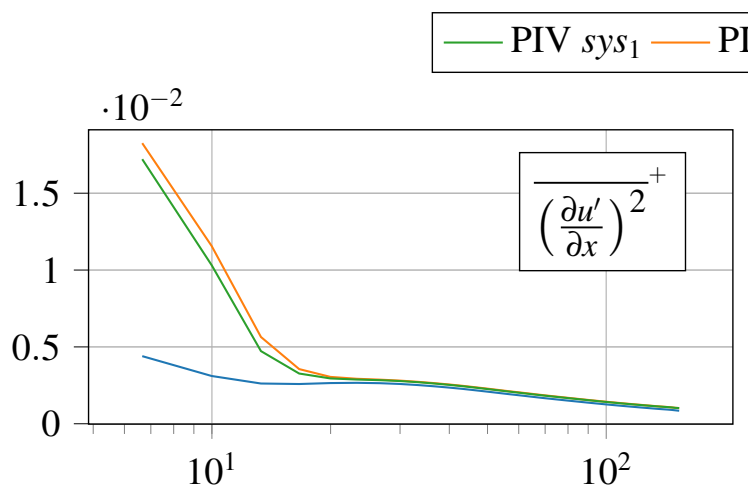

PIV sys 2 — PIV denoised

Figure 7: Effect of denoise on the derivatives of streamwise and wall-normal velocities at $R e_{\tau}=2220$

\section{Conclusion}

A method to denoise the statistics of turbulent flow is proposed in the present paper. This method is based on the use of two independant PIV systems. It allows the computation of the noise level of the order of 0.06 px. The derivative tends to amplify the noise (see Foucaut and Stanislas (2002)). This method allows also to denoise variances of velocity derivatives such as the terms of the dissipation rate. By using two different spatial resolutions we evidence that if the interrogation window size is of the order of $8 \mathrm{px}$ it is possible to compute these dissipation with a very good accuracy. If the interrogation window size increases (doubled here) the filtering effect of PIV is strongly observed in this parameter. If the interrogation window size is smaller than 4 Kolmogorov length scale which is about 2 wall units, the dissipation will be computed with a good accuracy.

\section{Acknowledgements}

This work was carried out within the framework of the CNRS Research Foundation on Ground Transport and Mobility, in articulation with the ELSAT2020 project supported by the European Community, the French Ministry of Higher Education and Research, and the Hauts de France Regional Council.

\section{References}

Adrian RJ, Meinhart CD, and Tomkins CD (2000) Vortex organization in the outer region of the turbulent boundary layer. Journal of fluid Mechanics 422:1-54

Atkinson C, Buchmann NA, Amili O, and Soria J (2014) On the appropriate filtering of piv measurements of turbulent shear flows. Experiments in fluids 55:1-15

Borrell G, Sillero JA, and Jiménez J (2013) A code for direct numerical simulation of turbulent boundary layers at high reynolds numbers in bg/p supercomputers. Computers \& Fluids 80:37-43

Foucaut JM, Carlier J, and Stanislas M (2004) Piv optimization for the study of turbulent flow using spectral analysis. Measurement Science and Technology 15:1046

Foucaut JM, Coudert S, Stanislas M, and Delville J (2011) Full 3d correlation tensor computed from double field stereoscopic piv in a high reynolds number turbulent boundary layer. Experiments in fluids 50:839846

Foucaut JM, George WK, Stanislas M, and Cuvier C (2020) Velocity derivatives in a high reynolds number turbulent boundary layer. part iii: Optimization of an spiv experiment for derivative moments assessment. arXiv preprint arXiv:201009364 
Foucaut JM and Stanislas M (2002) Some considerations on the accuracy and frequency response of some derivative filters applied to particle image velocimetry vector fields. Measurement Science and Technology 13:1058

George WK and Hussein HJ (1991) Locally axisymmetric turbulence. Journal of Fluid Mechanics 233:1-23

George WK and Stanislas M (2020) On the noise in statistics of piv measurements. arXiv preprint arXiv:201010768

Herpin S, Stanislas M, Foucaut JM, and Coudert S (2013) Influence of the reynolds number on the vortical structures in the logarithmic region of turbulent boundary layers. Journal of Fluid Mechanics 716:5

Hoyas S and Jiménez J (2008) Reynolds number effects on the reynolds-stress budgets in turbulent channels. Physics of Fluids 20:101511

Wieneke B (2008) Volume self-calibration for 3d particle image velocimetry. Experiments in fluids 45:549556 